\title{
Existence of Positive Solutions for a class of Quasilinear Schrödinger Equations of Choquard type *
}

\author{
Shaoxiong Chen ${ }^{\dagger}$ and $\mathrm{Xian} \mathrm{Wu}^{\ddagger}$ \\ Department of Mathematics, Yunnan Normal University, Kunming, Yunnan 650092, P. R. China
}

\begin{abstract}
In this paper, we study the following quasilinear Schrödinger equation of Choquard type

$$
-\triangle u+V(x) u-\triangle\left(u^{2}\right) u=\left(I_{\alpha} *|u|^{p}\right)|u|^{p-2} u, \quad x \in \mathbb{R}^{N},
$$

where $N \geq 3,0<\alpha<N, \frac{2(N+\alpha)}{N} \leq p<\frac{2(N+\alpha)}{N-2}$ and $I_{\alpha}$ is a Riesz potential. Under appropriate assumptions on $V(x)$, we establish the existence of positive solutions.
\end{abstract}

Key Words: quasilinear Schrödinger equations, Choquard type, Variational methods.

\section{Introduction and Preliminaries}

Consider the following quasilinear Schrödinger equation of Choquard type

$$
-\triangle u+V(x) u-\triangle\left(u^{2}\right) u=\left(I_{\alpha} *|u|^{p}\right)|u|^{p-2} u, \quad x \in \mathbb{R}^{N},
$$

where $N \geq 3,0<\alpha<N, \frac{2(N+\alpha)}{N} \leq p<\frac{2(N+\alpha)}{N-2}, V \in C\left(\mathbb{R}^{N}, \mathbb{R}\right)$ and $I_{\alpha}: \mathbb{R}^{N} \rightarrow \mathbb{R}$ is the Riesz potential defined by

$$
I_{\alpha}(x)=\frac{\Gamma\left(\frac{N-\alpha}{2}\right)}{\Gamma\left(\frac{\alpha}{2}\right) \pi^{N / 2} 2^{\alpha}|x|^{N-\alpha}}:=\frac{A_{\alpha}}{|x|^{N-\alpha}},
$$

${ }^{*}$ This work was supported partially by the National Natural Science Foundation of China (11562021).

†gxmail@126. com

${ }^{\ddagger}$ Corresponding Author: wuxian2042@163. com 
and $\Gamma$ is the Gamma function.

Eq.(1.1) is related to quasilinear Schrödinger equations of more general form

$$
i \psi_{t}+\triangle \psi-V(x) \psi+k \triangle\left(h\left(|\psi|^{2}\right)\right) h^{\prime}\left(|\psi|^{2}\right) \psi+g(x, \psi)=0
$$

where $V=V(x), x \in R^{N}$, is a given potential, $k$ is a real constant and $h, g$ are real functions. The quasilinear schrödinger equations (1.2) are derived as models of several physical phenomena, such as see [10, 11, 13, 19, 22. It has received considerable attention in mathematical analysis during the last 10 years [20]. When $k \neq 0$ and $g$ is a local term, several methods can be used to solve Eq. (1.2). The existence of a positive ground state solution has been proved in [14, 21] by using a constrained minimization approach. The problem is transformed to a semilinear one in [4, 15, 5] by a change of variables. Nehari method is used to get the existence results of ground state solutions in [16, 23].

For $k=0, V(x) \equiv 1$ and $g(x, \psi)=\left(I_{\alpha} *|\psi|^{p}\right)|\psi|^{p-2} \psi$, Eq.(1.2) is usually called the nonlinear Choquard or Choquard-Pekar equation. It has several physical origins. The problem appeared at least as early as in 1954, in a work by S. I. Pekar describing the quantum mechanics of a polaron at rest. In 1976, for $N=3, \alpha=2, \mathrm{P}$. Choquard used it to describe an electron trapped in its own hole, in a certain approximation to Hartree-Fock theory of one component plasma(see [17]). In that case, a ground state solution is found in [17]. For $p \in\left(\frac{N+\alpha}{N}, \frac{N+\alpha}{N-2}\right)$, Moroz and Van Schaftingen in [17] proved existence, qualitative properties and decay estimates of ground state solutions. In [24], Seok consider a critical version of nonlinear Choquard equation

$$
-\triangle u+u=\left(I_{\alpha} *|u|^{p}\right)|u|^{p-2} u+\lambda|u|^{2^{*}-2} u, \quad x \in \mathbb{R}^{N} .
$$

Using some perturbation arguments, Seok gets a family of nontrivial solutions. It converges to a least energy solution of the limiting critical local problem as $\alpha \rightarrow 0$. We can see some works of literature about the above equation in [1, 12, 28].

In this paper, we study the existence of positive solution of (1.1) by a variational argument. We need the following several notations. If $x \in \mathbb{R}^{N}$ and $R>0$, the closed ball with center at $x$ and radius $R$ is always denoted by $B_{R}(x)$. Let $C_{0}^{\infty}\left(\mathbb{R}^{N}\right)$ be the collection of 
smooth functions with compact support. For $N \geq 3$, let

$$
D^{1,2}\left(\mathbb{R}^{N}\right):=\left\{u \in L^{2^{*}}\left(\mathbb{R}^{N}\right): \nabla u \in L^{2}\left(\mathbb{R}^{N}\right)\right\}
$$

with the norm

$$
\|u\|_{D^{1,2}}^{2}=\int_{\mathbb{R}^{N}}|\nabla u|^{2} .
$$

By the Sobolev inequality, $D^{1,2}\left(\mathbb{R}^{N}\right)$ is continuously embedded in $L^{2^{*}}\left(\mathbb{R}^{N}\right)$. Let

$$
H^{1}\left(\mathbb{R}^{N}\right):=\left\{u \in L^{2}\left(\mathbb{R}^{N}\right): \nabla u \in L^{2}\left(\mathbb{R}^{N}\right)\right\}
$$

with the inner product

$$
\langle u, v\rangle_{H^{1}}=\int_{\mathbb{R}^{N}}(\nabla u \cdot \nabla v+u v)
$$

and the norm

$$
\|u\|:=\|u\|_{H^{1}}=\langle u, u\rangle_{H^{1}}^{1 / 2} .
$$

We denote the norm of $L^{q}\left(\mathbb{R}^{N}\right)$ by $|\cdot|_{q}$.

In the following, we always assume $V \in C\left(\mathbb{R}^{N}, \mathbb{R}\right)$ and $\inf _{\mathbb{R}^{N}} V(x) \geq V_{0}>0$. Let us consider the following two assumptions:

$\left(V_{1}\right) \quad V(x)$ is periodic in each variable of $x_{1}, \cdots, x_{N}$.

$\left(V_{2}\right) \quad V(x) \leq V_{\infty}:=\lim _{|y| \rightarrow \infty} V(y)<\infty$ for all $x \in \mathbb{R}^{N}$ and $V_{0}<V_{\infty}$.

Eq. (1.1) is the Euler-Lagrange equation of the energy functional

$$
J(u)=\frac{1}{2} \int_{\mathbb{R}^{N}}\left[\left(1+2 u^{2}\right)|\nabla u|^{2}+V(x) u^{2}\right]-\frac{1}{2 p} \int_{\mathbb{R}^{N}}\left(I_{\alpha} *\left|u^{+}\right|^{p}\right)\left|u^{+}\right|^{p},
$$

where $u^{+}=\max \{u, 0\}$.

The main result of this paper is stated as follows:

Theorem 1.1. Suppose that $N \geq 3, \frac{2(N+\alpha)}{N} \leq p<\frac{2(N+\alpha)}{N-2}$ and the potential function $V$ satisfies condition $\left(V_{1}\right)$ or $\left(V_{2}\right)$. Then Eq. (1.1) possesses a positive solution $u \in H^{1}\left(\mathbb{R}^{N}\right)$.

Remark 1.2. By the Hardy-Littlewood-Sobolev inequality and the Sobolev embedding theorem, the natural interval for considering the Choquard equation is $\left[\frac{2(N+\alpha)}{N}, \frac{2(N+\alpha)}{N-2}\right]$, however, the critical case $p=\frac{2(N+\alpha)}{N-2}$ is not considered in Theorem 1.1. 
To deal with this type of problem, difficulties lie in two aspects. First, the approach of proving Theorem 1.1 is inspired by [4, 6, 8, 15]. Because the nonlinearity of Eq. (1.1) is nonlocal, the techniques in these papers developed for the local case cannot be adopted directly. Second, another major difficulty here is that the energy functional $J(u)$ is not well defined for all $u \in H^{1}\left(\mathbb{R}^{N}\right)$ if $N \geq 3$. To overcome these difficulties, we need to make a change $u=f(v)$ of variable used in [15], to analyze other properties of $f$ more deeply due to the nonlocal nonlinearity and to develop some different techniques.

Following the idea in [15], let $f$ be defined by

$$
f^{\prime}(t)=\frac{1}{\sqrt{1+2 f^{2}(t)}}
$$

on $[0,+\infty), f(0)=0$ and $f(-t)=-f(t)$ on $(-\infty, 0]$. Then $f$ has following properties (see [3]):

$\left(f_{1}\right) \quad f$ is uniquely defined $C^{\infty}$ function and invertible.

$\left(f_{2}\right) \quad 0<f^{\prime}(t) \leq 1$ for all $t \in \mathbb{R}$.

$\left(f_{3}\right) \quad|f(t)| \leq|t|$ for all $t \in \mathbb{R}$.

$\left(f_{4}\right) \frac{1}{2} f(t) \leq t f^{\prime}(t) \leq f(t)$ for all $t \geq 0$ and $f(t) \leq t f^{\prime}(t) \leq \frac{1}{2} f(t)$ for all $t \leq 0$.

$\left(f_{5}\right)|f(t)| \leq 2^{\frac{1}{4}}|t|^{\frac{1}{2}}$ for all $t \in \mathbb{R}$.

$\left(f_{6}\right)$ There exists a positive constant $C$ such that

$$
|f(t)| \geq \begin{cases}C|t|, & |t| \leq 1 \\ C|t|^{\frac{1}{2}}, & |t| \geq 1\end{cases}
$$

$\left(f_{7}\right)\left|f(t) f^{\prime}(t)\right| \leq \frac{1}{\sqrt{2}}$ for all $t \in \mathbb{R}$.

$\left(f_{8}\right)$ For each $\xi>0$, there exists $C(\xi)>0$ such that $f^{2}(\xi t) \leq C(\xi) f^{2}(t)$.

After the change $u=f(v)$ of variable, Eq. (1.1) can be rewritten as

$$
-\triangle v+V(x) f(v) f^{\prime}(v)=\left(I_{\alpha} *|f(v)|^{p}\right)|f(v)|^{p-2} f(v) f^{\prime}(v), \quad x \in \mathbb{R}^{N},
$$


and $J(u)$ can be reduced to

$$
I(v):=\frac{1}{2} \int_{\mathbb{R}^{N}}\left(|\nabla v|^{2}+V(x) f^{2}(v)\right)-\frac{1}{2 p} \int_{\mathbb{R}^{N}}\left(I_{\alpha} *\left|f\left(v^{+}\right)\right|^{p}\right)\left|f\left(v^{+}\right)\right|^{p} .
$$

From $\left(V_{1}\right)$ (or $\left.\left(V_{2}\right)\right),\left(f_{3}\right),\left(f_{7}\right)$ and the Hardy-Littlewood-Sobolev inequality (Lemma 2.1), we can deduce that the functional $I \in C^{1}\left(H^{1}\left(\mathbb{R}^{N}\right)\right)$ for $\frac{2(N+\alpha)}{N} \leq p \leq \frac{2(N+\alpha)}{N-2}$.

It is easy to see that if $v \in H^{1}\left(\mathbb{R}^{N}\right)$ is a critical point of $I$, i.e.,

$$
\left\langle I^{\prime}(v), \varphi\right\rangle=\int_{\mathbb{R}^{N}} \nabla v \nabla \varphi+\int_{\mathbb{R}^{N}} V(x) f(v) f^{\prime}(v) \varphi-\int_{\mathbb{R}^{N}}\left(I_{\alpha} *\left|f\left(v^{+}\right)\right|^{p}\right)\left|f\left(v^{+}\right)\right|^{p-1} f^{\prime}\left(v^{+}\right) \varphi
$$

for all $\varphi \in C_{0}^{\infty}\left(\mathbb{R}^{N}\right)$, then $v$ is a weak solution of Eq. (1.3) and $u:=f(v)$ is a weak solution of Eq. (1.1).

We use $C$ or $C_{i}$ to denote various positive constants in context. The outline of the paper is as follows. In Section 2, we give some preliminary results and the regularity of solutions. In Section 3, we prove Theorem 1.1 by using the mountain pass theorem.

\section{Some lemmas}

Lemma 2.1 ([17]) (Hardy-Littlewood-Sobolev inequality). Let $r, s>1$ and $0<\alpha<N$ be such that

$$
\frac{1}{r}+\frac{1}{s}-\frac{\alpha}{N}=1
$$

Let $g \in L^{r}\left(\mathbb{R}^{N}\right)$ and $h \in L^{s}\left(\mathbb{R}^{N}\right)$. There exists a sharp constant $C(r, s, N, \alpha)$, independent of $g$, h, such that

$$
\int_{\mathbb{R}^{N}} \int_{\mathbb{R}^{N}} \frac{g(x) h(y)}{|x-y|^{N-\alpha}} \leq C(r, s, N, \alpha)|g|_{r}|h|_{s} .
$$

Remark 2.2 (1) Hardy-Littlewood-Sobolev inequality can be also stated that for every $s \in\left(1, \frac{N}{\alpha}\right)$, for every $v \in L^{s}\left(\mathbb{R}^{N}\right), I_{\alpha} * v \in L^{\frac{N s}{N-\alpha s}}\left(\mathbb{R}^{N}\right)$ and the following inequality holds:

$$
\int_{\mathbb{R}^{N}} \int_{\mathbb{R}^{N}}\left|I_{\alpha} * v\right|^{\frac{N s}{N-\alpha s}} \leq C\left(\int_{\mathbb{R}^{N}}|v|^{s}\right)^{\frac{N}{N-\alpha s}},
$$

where $C>0$ depends only on $\alpha, N$ and $s$. 
(2) By Lemma 2.1 and $\left(f_{5}\right)$,

$$
\int_{\mathbb{R}^{N}} \int_{\mathbb{R}^{N}} \frac{|f(v(x))|^{p}|f(v(y))|^{p}}{|x-y|^{N-\alpha}} \leq C_{1} \int_{\mathbb{R}^{N}} \int_{\mathbb{R}^{N}} \frac{|v(x)|^{\frac{p}{2}}|v(y)|^{\frac{p}{2}}}{|x-y|^{N-\alpha}} \leq C|v|_{\frac{p}{2} r}^{p}
$$

if $v \in L^{\frac{p}{2} r}\left(\mathbb{R}^{N}\right)$ for $r>1$ with

$$
\frac{2}{r}-\frac{\alpha}{N}=1
$$

Since we will work on $H^{1}\left(\mathbb{R}^{N}\right)$, by the Sobolev embedding theorem, we must require that $\frac{p}{2} r \in\left[2,2^{*}\right]$. We have

$$
\frac{2(N+\alpha)}{N} \leq p \leq \frac{2(N+\alpha)}{N-2}
$$

(3) $H^{1}\left(\mathbb{R}^{N}\right) \subset L^{\frac{2 N q}{N+\alpha}}\left(\mathbb{R}^{N}\right)$ if and only if $\frac{N+\alpha}{N} \leq q \leq \frac{N+\alpha}{N-2}$ where $q:=\frac{p}{2}$ (see [17]).

Lemma $2.3|f(t)| \leq 2^{\frac{1}{4}}|t|^{l}$ for all $l \in\left[\frac{1}{2}, 1\right], t \in \mathbb{R}$.

Proof. By $\left(f_{3}\right),\left(f_{5}\right)$, for $|t| \leq 1$,

$$
|f(t)| \leq|t| \leq|t|^{l} \leq 2^{\frac{1}{4}}|t|^{l}
$$

For $|t|>1$,

$$
|f(t)| \leq 2^{\frac{1}{4}}|t|^{\frac{1}{2}} \leq 2^{\frac{1}{4}}|t|^{l} .
$$

Lemma 2.4 For $N \geq 3,0<\alpha<N, \frac{2(N+\alpha)}{N} \leq p \leq \frac{2(N+\alpha)}{N-2}$, there exists $C>0$ and $r \in\left[2,2^{*}\right)$ such that

$$
|f(t)|^{p} \leq C t^{r},|f(t)|^{p-2} f(t) f^{\prime}(t) \leq C t^{r-1}, \quad \forall t \geq 0 .
$$

Proof. If $\frac{p}{2} \geq 2$, we put $r=\frac{p}{2}$. Since $p \leq \frac{2(N+\alpha)}{N-2}$ and $\alpha<N, r \in\left[2,2^{*}\right)$. By $\left(f_{5}\right),\left(f_{7}\right)$, we have

$$
|f(t)|^{p-2} f(t) f^{\prime}(t) \leq C t^{\frac{1}{2}(p-2)}=C t^{r-1}
$$

and

$$
|f(t)|^{p}=\left|f^{2}(t)\right|^{\frac{p}{2}} \leq C t^{r}
$$

for all $t \geq 0$. 
If $\frac{p}{2}<2$, by $2<\frac{2(N+\alpha)}{N} \leq p$ and Lemma 2.3, then there exists $l \in\left[\frac{1}{2}, 1\right]$ such that $r:=l p=2$. Hence

$$
|f(t)|^{p} \leq C|t|^{l p}=C t^{r}, \quad \forall t \geq 0 .
$$

Moreover, for $t \geq 1$, using $\left(f_{7}\right)$,

$$
|f(t)|^{p-2} f(t) f^{\prime}(t) \leq C t^{l(p-2)} \leq C t^{l(p-2)} \leq C t^{l p-1}=C t^{r-1} .
$$

For $0 \leq t<1$, by $\left(f_{2}\right)$ and $\left(f_{3}\right)$, we have

$$
|f(t)|^{p-2} f(t) f^{\prime}(t) \leq C t^{l(p-2)} t \leq C t^{l p-2} t=C t^{r-1}
$$

In the spirit of the argument developed by the Proposition 4.1 in [17] and the Lemma 2.1 in [2], we have the following Lemma 2.5.

Lemma 2.5 Assume that either assumption $\left(V_{1}\right)$ or $\left(V_{2}\right)$ holds. Let $N \geq 3,0<\alpha<$ $N, \frac{2(N+\alpha)}{N} \leq p<\frac{2(N+\alpha)}{N-2}, v \in H^{1}\left(\mathbb{R}^{N}\right)$ be a nontrivial and nonnegative weak solution of (1.3), then the following properties hold:

(1) For every $s \in \mathbb{R}$ with $\frac{\alpha}{N}\left(1-\frac{2}{p}\right)-\frac{2}{N}<\frac{1}{s}<1$, we have $v \in L_{\text {loc }}^{s}\left(\mathbb{R}^{N}\right)$.

(2) $I_{\alpha} *|f(v)|^{p} \in L_{l o c}^{\infty}\left(\mathbb{R}^{N}\right)$.

(3) $v \in W_{l o c}^{2, q}\left(\mathbb{R}^{N}\right)$ for every $q \in[2, \infty)$.

(4) For any $\lambda \in(0,1)$ such that

$$
\left(I_{\alpha} *|f(v)|^{p}\right)|f(v)|^{p-2} f(v) f^{\prime}(v) \in C_{l o c}^{0, \lambda}\left(\mathbb{R}^{N}\right) .
$$

(5) $v$ is of class $C^{1, \lambda}$ for every $\lambda \in(0,1)$ and $v>0$.

Proof. (1) We use an iterating argument (see [17]). Put $r:=\frac{p}{2}>1$, since $v \in H^{1}\left(\mathbb{R}^{N}\right)$, By Remark 2.2-(3), $v \in L^{\frac{2 N r}{N+\alpha}}\left(\mathbb{R}^{N}\right)$. Set $s_{0}=\underline{s}_{0}=\bar{s}_{0}:=\frac{2 N r}{N+\alpha}$ and

$$
\frac{1}{t}=\frac{r}{s_{0}}-\frac{\alpha}{N} \in(0,1) \text {. }
$$

We have $I_{\alpha} *|v|^{r} \in L^{t}\left(\mathbb{R}^{N}\right)$. Further, set

$$
\frac{1}{q_{0}}=\frac{2 r-1}{s_{0}}-\frac{\alpha}{N}=\frac{r-1}{s_{0}}+\frac{1}{t} \in(0,1) .
$$


We get $\left(I_{\alpha} *|v|^{r}\right)|v|^{r-1} \in L^{q_{0}}\left(\mathbb{R}^{N}\right)$. By $\left(f_{4}\right),\left(f_{5}\right),\left(f_{7}\right), v \geq 0$ and

$$
\left(I_{\alpha} *|f(v)|^{p}\right)|f(v)|^{p-2} f(v) f^{\prime}(v) \leq C\left(I_{\alpha} *|v|^{\frac{p}{2}}\right)|v|^{\frac{p-2}{2}}=C\left(I_{\alpha} *|v|^{r}\right)|v|^{r-1},
$$

one has

$$
\left(I_{\alpha} *|f(v)|^{p}\right)|f(v)|^{p-2} f(v) f^{\prime}(v) \in L^{q_{0}}\left(\mathbb{R}^{N}\right) .
$$

Set

$$
c(x)= \begin{cases}\frac{V(x) f(v(x)) f^{\prime}(v(x))}{v(x)}, & v(x) \neq 0, \\ 1, & v(x)=0 .\end{cases}
$$

Using $\left(f_{2}\right),\left(f_{3}\right),\left(V_{1}\right)$ (or $\left.\left(V_{2}\right)\right)$, we know that $c(x) \in L_{l o c}^{\infty}\left(\mathbb{R}^{N}\right)$ and $c(x) v=V(x) f(v) f^{\prime}(v)$.

Hence $v$ is a weak solution of the following equation

$$
-\triangle v+c(x) v=\left(I_{\alpha} *|f(v)|^{p}\right)|f(v)|^{p-2} f(v) f^{\prime}(v), \quad x \in \mathbb{R}^{N},
$$

and hence, $v \in W_{l o c}^{2, q_{0}}\left(\mathbb{R}^{N}\right)$ by the Theorem 9.1.4 in [27].

Notice that $\frac{2(N+\alpha)}{N} \leq p<\frac{2(N+\alpha)}{N-2}$. By the Sobolev embedding theorem, $v \in L_{l o c}^{s}\left(\mathbb{R}^{N}\right)$ provided

$$
\frac{2 r-1}{s_{0}}-\frac{\alpha+2}{N} \leq \frac{1}{s} \leq \frac{2 r-1}{s_{0}}-\frac{\alpha}{N}
$$

Furthermore,

$$
\frac{2 r-1}{\bar{s}_{0}}-\frac{\alpha+2}{N}<\frac{1}{\bar{s}_{0}}=\frac{1}{s_{0}}=\frac{1}{\underline{s}_{0}} \leq \frac{2 r-1}{\underline{s}_{0}}-\frac{\alpha}{N} .
$$

Set $\frac{1}{\underline{s}_{1}}=\frac{2 r-1}{\underline{s}_{0}}-\frac{\alpha}{N}$ and $\frac{1}{\bar{s}_{1}}=\frac{2 r-1}{\bar{s}_{0}}-\frac{\alpha+2}{N}$. For every $s \in\left(\underline{s}_{1}, \bar{s}_{1}\right)$, we have $v \in L_{l o c}^{s}\left(\mathbb{R}^{N}\right)$. Then we can prove $v \in W_{l o c}^{2, q}\left(\mathbb{R}^{N}\right)$ provided

$$
\frac{2 r-1}{\bar{s}_{1}}-\frac{\alpha}{N}<\frac{1}{q}<\frac{2 r-1}{\underline{s}_{1}}-\frac{\alpha}{N}
$$

and

$$
\frac{\alpha}{N}\left(1-\frac{1}{r}\right)<\frac{1}{q}<1
$$

In fact, for every $s$ with $\frac{1}{q}=\frac{2 r-1}{s}-\frac{\alpha}{N}$, the above inequalities follows that

$$
\frac{\alpha}{N r}<\frac{1}{s}<\frac{N+\alpha}{N(2 r-1)}
$$

and

$$
s \in\left(\underline{s}_{1}, \bar{s}_{1}\right) .
$$


Hence

$$
\frac{1}{t}=\frac{r}{s}-\frac{\alpha}{N} \in(0,1)
$$

and hence $I_{\alpha} *|v|^{r} \in L_{l o c}^{t}\left(\mathbb{R}^{N}\right)$. Further, since

$$
\frac{1}{q}=\frac{2 r-1}{s}-\frac{\alpha}{N}=\frac{r-1}{s}+\frac{1}{t} \in(0,1)
$$

one has $\left(I_{\alpha} *|v|^{r}\right)|v|^{r-1} \in L_{l o c}^{q}\left(\mathbb{R}^{N}\right)$. Similar to the above segment, we can prove $v \in$ $W_{l o c}^{2, q}\left(\mathbb{R}^{N}\right)$.

Set

$$
\left(\frac{1}{\bar{q}_{1}}, \frac{1}{\underline{q}_{1}}\right)=\left(\frac{2 r-1}{\bar{s}_{1}}-\frac{\alpha}{N}, \frac{2 r-1}{\underline{s}_{1}}-\frac{\alpha}{N}\right) \cap\left(\frac{\alpha}{N}\left(1-\frac{1}{r}\right), 1\right)(\neq \emptyset),
$$

If $\frac{1}{\bar{q}_{1}}=\frac{\alpha}{N}\left(1-\frac{1}{r}\right)$ and $\frac{1}{\underline{q}_{1}}=\frac{2 r-1}{\underline{s}_{1}}-\frac{\alpha}{N}<1$, we use $\underline{q}_{1}$ in replace of $q_{0}$ in the above argument. Similarly, if $\frac{1}{\bar{q}_{1}}=\frac{2 r-1}{\bar{s}_{1}}-\frac{\alpha}{N}>\frac{\alpha}{N}\left(1-\frac{1}{r}\right)$ and $\frac{1}{\underline{q}_{1}}=1$, we use $\bar{q}_{1}$ in replace of $q_{0}$. So, without loss of generality, we can assume that $\frac{\alpha}{N}\left(1-\frac{1}{r}\right)<\frac{2 r-1}{\bar{s}_{1}}-\frac{\alpha}{N}$ and $\frac{2 r-1}{\underline{s}_{1}}-\frac{\alpha}{N}<1$ are satisfied. Hence, for every $q \in \mathbb{R}$ with $\frac{2 r-1}{\bar{s}_{1}}-\frac{\alpha}{N}<\frac{1}{q}<\frac{2 r-1}{\underline{s}_{1}}-\frac{\alpha}{N}$, we know that $v \in W_{l o c}^{2, q}\left(\mathbb{R}^{N}\right)$.

By the Sobolev embedding theorem again , $v \in L_{l o c}^{s}\left(\mathbb{R}^{N}\right)$ provided

$$
\frac{2 r-1}{\bar{s}_{1}}-\frac{\alpha+2}{N} \leq \frac{1}{s} \leq \frac{2 r-1}{\underline{s}_{1}}-\frac{\alpha}{N} \text {. }
$$

We also have

$$
\frac{\alpha}{N}\left(1-\frac{2}{p}\right)-\frac{2}{N}<\frac{1}{s}<1
$$

Furthermore, by $\frac{2(N+\alpha)}{N} \leq p<\frac{2(N+\alpha)}{N-2}$,

$$
\frac{2 r-1}{\bar{s}_{1}}-\frac{\alpha+2}{N}<\frac{1}{\bar{s}_{1}}<\frac{1}{\bar{s}_{0}}=\frac{1}{s_{0}}=\frac{1}{\underline{s}_{0}} \leq \frac{1}{\underline{s}_{1}} \leq \frac{2 r-1}{\underline{s}_{1}}-\frac{\alpha}{N} .
$$

Set $\frac{1}{\underline{s}_{2}}=\frac{2 r-1}{\underline{s}_{1}}-\frac{\alpha}{N}$ and $\frac{1}{\bar{s}_{2}}=\frac{2 r-1}{\bar{s}_{1}}-\frac{\alpha+2}{N}$. Then, for each $s \in\left(\underline{s}_{2}, \bar{s}_{2}\right)$, we have $v \in L_{l o c}^{s}\left(\mathbb{R}^{N}\right)$.

Continue the above process by setting

$$
\frac{1}{\bar{s}_{k+1}}=\frac{2 r-1}{\bar{s}_{k}}-\frac{\alpha+2}{N}, \frac{1}{\underline{s}_{k+1}}=\frac{2 r-1}{\underline{s}_{k}}-\frac{\alpha}{N} .
$$

Notice that

$$
\frac{1}{\bar{s}_{k+1}}-\frac{1}{\bar{s}_{k}}=(2 r-1)^{k}\left(\frac{1}{\bar{s}_{1}}-\frac{1}{\bar{s}_{0}}\right)<0, \frac{1}{\underline{s}_{k+1}}-\frac{1}{\underline{s}_{k}}=(2 r-1)^{k}\left(\frac{1}{\underline{s}_{1}}-\frac{1}{\underline{s}_{0}}\right) \geq 0 .
$$


If $r=p / 2=\frac{N}{N+\alpha}$, then $\frac{1}{\underline{s}_{k+1}}=\frac{1}{\underline{s}_{k}}=\cdots=\frac{1}{\underline{s}_{0}}=\frac{1}{s}<1$. If $\frac{2(N+\alpha)}{N}<p<\frac{2(N+\alpha)}{N-2}$, then $\frac{1}{\underline{s}_{1}}-\frac{1}{\underline{s}_{0}}>0$. Since $r>1$, there exists $k_{0} \geq 1$ such that

$$
\frac{1}{\bar{s}_{k}}<\frac{\alpha}{N}\left(1-\frac{2}{p}\right)-\frac{2}{N}<1<\frac{1}{\underline{s}_{k}} .
$$

Hence (1) holds.

(2) Since $p<\frac{2(N+\alpha)}{N-2}$,

$$
\frac{\alpha}{N}\left(1-\frac{2}{p}\right)-\frac{2}{N}<\frac{\alpha^{2}-2 N}{N(N+\alpha)}<\frac{\alpha^{2}-2 \alpha}{N(N+\alpha)}<\frac{2 \alpha}{N p} .
$$

Fix $\frac{1}{q} \in\left(\frac{\alpha}{N}\left(1-\frac{2}{p}\right)-\frac{2}{N}, \frac{2 \alpha}{N p}\right)$. It implies that $v \in L_{l o c}^{q}\left(\mathbb{R}^{N}\right)$ and $N-1+\frac{2 q}{2 q-p}(\alpha-N)>-1$. By virtue of the Lemma 2.4 and the Hölder's inequality, for every closed ball $B \subset \mathbb{R}^{N}$, there exists $r \in\left[2,2^{*}\right)$ such that

$$
\begin{aligned}
\left(I_{\alpha} *|f(v)|^{p}\right)(x) & =A_{\alpha}\left(\int_{|x-y|>1} \frac{|f(v(y))|^{p}}{|x-y|^{N-\alpha}} d y+\int_{|x-y| \leq 1} \frac{|f(v(y))|^{p}}{|x-y|^{N-\alpha}} d y\right) \\
& \leq C_{1}\left(\int_{|x-y|>1} \frac{|v(y)|^{r}}{|x-y|^{N-\alpha}} d y+\int_{|x-y| \leq 1} \frac{|f(v(y))|^{p}}{|x-y|^{N-\alpha}} d y\right) \\
& \leq C_{1} \int_{|x-y|>1}|v(y)|^{r} d y+C_{3} \int_{|x-y| \leq 1} \frac{|v(y)|^{\frac{p}{2}}}{|x-y|^{N-\alpha}} d y \\
& \leq C_{2}+C_{3}\left(\int_{|x-y| \leq 1}|x-y|^{(\alpha-N) \cdot \frac{2 q}{2 q-p}} d y\right)^{1-\frac{p}{2 q}}\left(\int_{|x-y| \leq 1}|v(y)|^{\frac{p}{2} \cdot \frac{2 q}{p}} d y\right)^{\frac{p}{2 q}} \\
& \leq C
\end{aligned}
$$

for all $x \in B$. That is $I_{\alpha} *|f(v)|^{p} \in L_{l o c}^{\infty}\left(\mathbb{R}^{N}\right)$.

(3) For every closed ball $B \subset \mathbb{R}^{N}$, set

$$
a(x)=\left|\frac{\left(I_{\alpha} *|f(v)|^{p}\right)|f(v)|^{p-2} f(v) f^{\prime}(v)-V(x) f(v) f^{\prime}(v)}{1+|v|}\right|, \forall x \in B .
$$

By the Lemma 2.4, the conclusion $(2),\left(V_{1}\right)$ or $\left(V_{2}\right)$, it follows that

$$
a(x) \leq V(x)+C_{1} \frac{|f(v)|^{p-2} f(v) f^{\prime}(v)}{1+|v|} \leq C_{2}+C_{1}|v|^{r-2}, \forall x \in B .
$$


Since

$$
\begin{aligned}
\int_{B}|v|^{(r-2) \cdot \frac{N}{2}} d x & =\int_{B \cap\{|v|<1 \mid\}}|v|^{(r-2) \cdot \frac{N}{2}} d x+\int_{B \cap\{|v| \geq 1\}}|v|^{(r-2) \cdot \frac{N}{2}} d x \\
& \leq C_{1}+\int_{B \cap\{|v| \geq 1\}}|v|^{\left(\frac{2 N}{N-2}-2\right) \cdot \frac{N}{2}} d x \\
& \leq C
\end{aligned}
$$

for all $x \in B, a(x) \in L^{\frac{N}{2}}(B)$. Hence $a(x) \in L_{l o c}^{\frac{N}{2}}\left(\mathbb{R}^{N}\right)$. Apply the Lemma B.3 in 25] to the following equation

$$
-\Delta v=a(x) v
$$

It follows that $v \in L_{l o c}^{q}\left(\mathbb{R}^{N}\right)$ for any $q \in[2,+\infty)$. Hence, by the Theorem 9.1.4 of [27], $v \in W_{l o c}^{2, q}\left(\mathbb{R}^{N}\right)$ for any $q \in[2,+\infty)$.

(4) From (3) and the Sobolev embedding theorem, we have that $v \in C_{l o c}^{1, \lambda}\left(\mathbb{R}^{N}\right)$ for any $\lambda \in(0,1)$. Fix $R>0$. Take $\beta \in C_{0}^{\infty}\left(\mathbb{R}^{N}\right)$ such that $\beta(t)=1$ for $t \leq R, \beta(t)=0$ for $t \geq 2 R$. Write $I_{\alpha}=\beta I_{\alpha}+(1-\beta) I_{\alpha}$. Put

$$
g(x)= \begin{cases}0, & |x| \leq R \\ (1-\beta(x)) I_{\alpha}(x), & |x| \geq R\end{cases}
$$

Then $g(x) \in C_{0}^{\infty}\left(\mathbb{R}^{N}\right)$. By the Lemma 2.4, there exists $r \in\left[2,2^{*}\right)$ such that

$$
g^{\prime}(x-\cdot)|f(v(\cdot))|^{p} \leq C|v(\cdot)|^{r} \in L^{1}\left(\mathbb{R}^{N}\right)
$$

for every $x \in \mathbb{R}^{N}$. Using the Lebesgue's dominated convergence theorem, we have

$$
\left(\left((1-\beta) I_{\alpha}\right) *|f(v)|^{p}\right)(x)=\int_{\mathbb{R}^{N}} g(x-y)|f(v(y))|^{p} d y \in C^{\infty}\left(\mathbb{R}^{N}\right) .
$$

It follows from $p>2, f \in C^{\infty}(\mathbb{R})$ that $|f(v)|^{p},|f(v)|^{p-2} f(v) f^{\prime}(v) \in C_{l o c}^{0, \lambda}\left(\mathbb{R}^{N}\right)$. It is easy to see that $\beta I_{\alpha} \in L^{1}\left(\mathbb{R}^{N}\right)$. Hence

$$
\left(\beta I_{\alpha}\right) *|f(v)|^{p} \in C_{l o c}^{0, \lambda}\left(\mathbb{R}^{N}\right)
$$

Therefore,

$$
\left(I_{\alpha} *|f(v)|^{p}\right)|f(v)|^{p-2} f(v) f^{\prime}(v) \in C_{l o c}^{0, \lambda}\left(\mathbb{R}^{N}\right) .
$$


(5) It follows from (4) that $v \in C_{l o c}^{1, \lambda}\left(\mathbb{R}^{N}\right)$ for every $\lambda \in(0,1)$. In the proof of conclusion (1), Eq. (1.3) can be rewritten as

$$
-\triangle v+c(x) v=\left(I_{\alpha} *|f(v)|^{p}\right)|f(v)|^{p-2} f(v) f^{\prime}(v) .
$$

Since $v$ is a continuous nonnegative function, there exists $C>0$ such that $|c(x)| \leq C$. Using the Theorem 8.19 in [9], we get that $v>0$ in $\mathbb{R}^{N}$.

\section{Proof of Theorem 1.1}

We prove that the functional $I$ exhibits the mountain pass geometry.

Lemma 3.1. There exist $\rho_{0}, \alpha>0$ such that

$$
I(v) \geq \alpha, \quad \forall v \in\left\{v \in H^{1}\left(\mathbb{R}^{N}\right):\|v\|=\rho_{0}\right\}
$$

Proof. From [8] we get that there exist $C_{1}>0, \rho_{1}>0$ such that

$$
\int_{\mathbb{R}^{N}}\left(|\nabla v|^{2}+V(x) f^{2}(v)\right) \geq C_{1}\|v\|^{2}
$$

whenever $\|v\| \leq \rho_{1}$. The above inequality was derived in [8] for $\left(V_{1}\right)$. Checking the proof of [8], we know that this inequality holds for $\left(V_{2}\right)$, too. Notice that $\frac{N p}{N+\alpha} \in\left[2,2^{*}\right)$. By $\left(f_{5}\right)$, (2.1), (3.1) and the Sobolev embedding theorem, we get

$$
\begin{aligned}
I(v) & \geq \frac{C_{1}}{2}\|v\|^{2}-\frac{1}{2 p} \int_{\mathbb{R}^{N}}\left(I_{\alpha} *\left|f\left(v^{+}\right)\right|^{p}\right)\left|f\left(v^{+}\right)\right|^{p} \\
& \geq \frac{C_{1}}{2}\|v\|^{2}-C_{2} \int_{\mathbb{R}^{N}}\left(I_{\alpha} *|v|^{p / 2}\right)|v|^{p / 2} \\
& \geq \frac{C_{1}}{2}\|v\|^{2}-C_{2}\left(\int_{\mathbb{R}^{N}}|v|^{\frac{N p}{N+\alpha}}\right)^{\frac{N+\alpha}{N}} \\
& \geq \frac{C_{1}}{2}\|v\|^{2}-C_{3}\|v\|^{p} \\
& \geq\|v\|^{2}\left(\frac{C_{1}}{2}-C_{3}\|v\|^{p-2}\right)
\end{aligned}
$$

whenever $\|v\| \leq \rho_{1}$. Choosing $\rho_{0}$ small enough, we get the proof.

Using the method in [6], we have the following lemma: 
Lemma 3.2. There exists $v_{0} \in H^{1}\left(\mathbb{R}^{N}\right)$ such that $\left\|v_{0}\right\|>\rho_{0}$ and $I\left(v_{0}\right)<0$.

Proof. By $\left(f_{4}\right), \frac{f(t)}{t}$ is decreasing for $t>0$. Consider $\phi \in C_{0}^{\infty}\left(\mathbb{R}^{N}\right)$ such that $0 \leq$ $\phi(x) \leq 1, \phi(x)=1$ for $|x| \leq 1, \phi(x)=0$ for $|x| \geq 2$. We have

$$
f(t \phi(x)) \geq f(t) \phi(x)
$$

for any $x \in \mathbb{R}^{N}, t>0$. Using $\left(f_{3}\right)$, we get

$$
\begin{aligned}
I(t \phi) & =\frac{t^{2}}{2} \int_{\mathbb{R}^{N}}|\nabla \phi|^{2}+\frac{1}{2} \int_{\mathbb{R}^{N}} V(x) f^{2}(t \phi)-\frac{1}{2 p} \int_{\mathbb{R}^{N}}\left(I_{\alpha} *|f(t \phi)|^{p}\right)|f(t \phi)|^{p} \\
& \leq \frac{t^{2}}{2} \int_{\mathbb{R}^{N}}|\nabla \phi|^{2}+\frac{t^{2}}{2} \int_{\mathbb{R}^{N}} V(x) \phi^{2}-\frac{1}{2 p} f^{2 p}(t) \int_{\mathbb{R}^{N}}\left(I_{\alpha} *|\phi|^{p}\right)|\phi|^{p} \\
& \leq \frac{t^{2}}{2}\left(C_{1}\|\phi\|^{2}-C_{2} \frac{f^{4}(t)}{t^{2}} \cdot f^{2 p-4}(t)\right) .
\end{aligned}
$$

By $p>2$ and $\left(f_{6}\right)$, we deduce that $I\left(t_{0} \phi\right)<0$ and $t_{0}\|\phi\|>\rho_{0}$ for $t_{0}$ large enough. Set $v_{0}=t_{0} \phi$. Hence $v_{0}$ is required.

Lemma 3.3. All Cerami sequences for $I$ at the level $c>0$ are bounded in $H^{1}\left(\mathbb{R}^{N}\right)$.

Proof. Let $\left(v_{n}\right) \subset H^{1}\left(\mathbb{R}^{N}\right)$ be a Cerami sequence at the level $c$. Set $w_{n}:=\frac{f\left(v_{n}\right)}{f^{\prime}\left(v_{n}\right)}$. It follows from $\left(f_{4}\right)$ that

$$
\begin{gathered}
\int_{\mathbb{R}^{N}}\left|w_{n}\right|^{2} \leq 4 \int_{\mathbb{R}^{N}}\left|v_{n}\right|^{2} \\
\int_{\mathbb{R}^{N}}\left|\nabla w_{n}\right|^{2}=\int_{\mathbb{R}^{N}}\left(1+\frac{2 f^{2}\left(v_{n}\right)}{1+2 f^{2}\left(v_{n}\right)}\right)^{2}\left|\nabla v_{n}\right|^{2} \leq 4 \int_{\mathbb{R}^{N}}\left|\nabla v_{n}\right|^{2},
\end{gathered}
$$

and

$$
\left|\left\langle I^{\prime}\left(v_{n}\right), w_{n}\right\rangle\right| \leq C\left\|I^{\prime}\left(v_{n}\right)\right\|\left(\left\|v_{n}\right\|+1\right) \rightarrow 0, \text { as } n \rightarrow \infty .
$$

It follows that $\left(w_{n}\right) \subset H^{1}\left(\mathbb{R}^{N}\right)$ is bounded. So

$$
\begin{aligned}
c+1 \geq & I\left(v_{n}\right)-\frac{1}{2 p}\left\langle I^{\prime}\left(v_{n}\right), w_{n}\right\rangle \\
= & \frac{1}{2} \int_{\mathbb{R}^{N}}\left|\nabla v_{n}\right|^{2}+\frac{1}{2} \int_{\mathbb{R}^{N}} V(x) f^{2}\left(v_{n}\right)-\frac{1}{2 p} \int_{\mathbb{R}^{N}}\left(I_{\alpha} *\left|f\left(v_{n}^{+}\right)\right|^{p}\right)\left|f\left(v_{n}^{+}\right)\right|^{p} \\
& -\frac{1}{2 p} \int_{\mathbb{R}^{N}}\left(1+\frac{2 f^{2}\left(v_{n}\right)}{1+2 f^{2}\left(v_{n}\right)}\right)\left|\nabla v_{n}\right|^{2}-\frac{1}{2 p} \int_{\mathbb{R}^{N}} V(x) f^{2}\left(v_{n}\right) \\
& +\frac{1}{2 p} \int_{\mathbb{R}^{N}}\left(I_{\alpha} *\left|f\left(v_{n}^{+}\right)\right|^{p}\right)\left|f\left(v_{n}^{+}\right)\right|^{p} \\
\geq & \left(\frac{1}{2}-\frac{1}{p}\right)\left(\int_{\mathbb{R}^{N}}\left|\nabla v_{n}\right|^{2}+\int_{\mathbb{R}^{N}} V(x) f^{2}\left(v_{n}\right)\right) .
\end{aligned}
$$


Since $p>2$, the sequence $\left\{\int_{\mathbb{R}^{N}}\left|\nabla v_{n}\right|^{2}+\int_{\mathbb{R}^{N}} V(x) f^{2}\left(v_{n}\right)\right\}$ is bounded. By the Sobolev embedding theorem and $\left(f_{6}\right)$, we have

$$
\begin{aligned}
\int_{\mathbb{R}^{N}}\left|v_{n}\right|^{2} & =\int_{\{|v| \leq 1\}}\left|v_{n}\right|^{2}+\int_{\{|v|>1\}}\left|v_{n}\right|^{2} \\
& \leq C_{1} \int_{\{|v| \leq 1\}}\left|f\left(v_{n}\right)\right|^{2}+\left(\int_{\{|v|>1\}}\left|v_{n}\right|\right)^{\theta}\left(\int_{\{|v|>1\}}\left|v_{n}\right|^{2^{*}}\right)^{1-\theta} \\
& \leq C_{1} \int_{\mathbb{R}^{N}} f^{2}\left(v_{n}\right)+\left(\int_{\{|v|>1\}} f^{2}\left(v_{n}\right)\right)^{\theta}\left(\int_{\mathbb{R}^{N}}\left|v_{n}\right|^{2^{*}}\right)^{1-\theta} \\
& \leq C_{2} \int_{\mathbb{R}^{N}} V(x) f^{2}\left(v_{n}\right)+C_{3}\left(\int_{\mathbb{R}^{N}} V(x) f^{2}\left(v_{n}\right)\right)^{\theta}\left(\int_{\mathbb{R}^{N}}\left|\nabla v_{n}\right|^{2}\right)^{(1-\theta) \frac{2^{*}}{2}} \\
& \leq C,
\end{aligned}
$$

where $\theta=\frac{2^{*}-2}{2\left(2^{*}-1\right)}$. Hence $\left(v_{n}\right)$ is bounded in $H^{1}\left(\mathbb{R}^{N}\right)$.

Lemma 3.4. Let $\Omega$ be a domain in $\mathbb{R}^{N}$. Suppose $\left\{g_{n}\right\},\left\{h_{n}\right\} \subset L^{1}(\Omega)$ and $h \in L^{1}(\Omega)$. If

$$
0 \leq g_{n} \leq h_{n}, \quad g_{n}(x) \rightarrow 0, \quad h_{n} \rightarrow h \text { a.e. } x \in \Omega
$$

and

$$
\lim _{n \rightarrow \infty} \int_{\Omega} h_{n}=\int_{\Omega} h
$$

then $\lim _{n \rightarrow \infty} \int_{\Omega} g_{n}=0$.

Proof. By Fatou's lemma,

$$
\begin{aligned}
\int_{\Omega} h & =\int_{\Omega} \liminf _{n \rightarrow \infty}\left(h_{n}-g_{n}\right) \\
& \leq \liminf _{n \rightarrow \infty} \int_{\Omega}\left(h_{n}-g_{n}\right) \\
& =\liminf _{n \rightarrow \infty} \int_{\Omega} h_{n}+\liminf _{n \rightarrow \infty} \int_{\Omega}\left(-g_{n}\right) \\
& =\int_{\Omega} h-\limsup _{n \rightarrow \infty} \int_{\Omega} g_{n} .
\end{aligned}
$$

Therefore, $0 \leq \liminf _{n \rightarrow \infty} \int_{\Omega} g_{n} \leq \limsup _{n \rightarrow \infty} \int_{\Omega} g_{n} \leq 0$, that is, $\lim _{n \rightarrow \infty} \int_{\Omega} g_{n}=0$.

In the following, we always assume that $\left\{v_{n}\right\} \subset H^{1}\left(\mathbb{R}^{N}\right)$ is a Cerami sequence for $I$ at the level $c>0$. By the preceding lemma, $\left\{v_{n}\right\}$ is bounded. We may assume, going if 
necessary to a subsequence, $v_{n} \rightarrow v \in H^{1}\left(\mathbb{R}^{N}\right), v_{n}(x) \rightarrow v(x)$ a.e. $x \in \mathbb{R}^{N}$ and $v_{n} \rightarrow v$ in $L_{l o c}^{q}\left(\mathbb{R}^{N}\right)$ for all $q \in\left[2,2^{*}\right)$. We have the following Lemma 3.5-3.8.

Lemma 3.5. If $\int_{\mathbb{R}^{N}}\left|f\left(v_{n}\right)\right|^{2} \rightarrow \int_{\mathbb{R}^{N}}|f(v)|^{2}$ as $n \rightarrow \infty$, then $\left\|v_{n}-v\right\| \rightarrow 0$.

Proof. The proof of Lemma 3.5 will be carried out in a series of steps.

Step 1. $\int_{\mathbb{R}^{N}} V(x)\left|f\left(v_{n}-v\right)\right|^{2} \rightarrow 0$ as $n \rightarrow \infty$.

By $\left(f_{3}\right),\left\{f\left(v_{n}\right)\right\}$ is bounded in $L^{2}\left(\mathbb{R}^{N}\right)$. We can assume $f\left(v_{n}\right) \rightarrow f(v)$ in $L^{2}\left(\mathbb{R}^{N}\right)$, and so $\int_{\mathbb{R}^{N}}\left|f\left(v_{n}\right)-f(v)\right|^{2} \rightarrow 0$. Therefore, by $\left(V_{1}\right)$ or $\left(V_{1}\right)$, one has

$$
\begin{aligned}
\left.\left|\int_{\mathbb{R}^{N}} V(x)\right| f\left(v_{n}\right)\right|^{2}-\int_{\mathbb{R}^{N}} V(x)|f(v)|^{2} \mid & \leq\left.\int_{\mathbb{R}^{N}} V(x)|| f\left(v_{n}\right)\right|^{2}-|f(v)|^{2} \mid \\
& \leq\left. V_{\infty} \int_{\mathbb{R}^{N}}|| f\left(v_{n}\right)\right|^{2}-|f(v)|^{2} \mid \rightarrow 0 .
\end{aligned}
$$

By $\left(f_{8}\right)$, there is $C>0$ such that $f^{2}(2 t) \leq C f^{2}(t)$. Since $f^{2}(t)$ is convex,

$$
\begin{aligned}
V(x) f^{2}\left(v_{n}-v\right) & \leq V(x) f^{2}\left(\frac{1}{2}\left(2 v_{n}-2 v\right)\right) \\
& \leq \frac{1}{2} V(x) f^{2}\left(2 v_{n}\right)+\frac{1}{2} V(x) f^{2}(-2 v) \\
& \leq \frac{1}{2} V(x) f^{2}\left(2 v_{n}\right)+\frac{1}{2} V(x) f^{2}(2 v) \\
& \leq C\left(V(x) f^{2}\left(v_{n}\right)+V(x) f^{2}(v)\right) .
\end{aligned}
$$

Using the Lemma 3.4,

$$
\lim _{n \rightarrow \infty} \int_{\mathbb{R}^{N}} V(x) f^{2}\left(v_{n}-v\right)=0
$$

Step 2. For any $q \in\left[2,2^{*}\right), \int_{\mathbb{R}^{N}}\left|v_{n}-v\right|^{q} \rightarrow 0$ as $n \rightarrow \infty$.

Check the proof of the Lemma 3.3. We have

$$
\begin{aligned}
& \int_{\mathbb{R}^{N}}\left|v_{n}-v\right|^{2} \\
\leq & C_{1} \int_{\mathbb{R}^{N}} V(x) f^{2}\left(v_{n}-v\right)+C_{2}\left(\int_{\mathbb{R}^{N}} V(x) f^{2}\left(v_{n}-v\right)\right)^{\theta}\left(\int_{\mathbb{R}^{N}}\left|\nabla\left(v_{n}-v\right)\right|^{2}\right)^{(1-\theta) \frac{2^{*}}{2}},
\end{aligned}
$$

where $\theta=\frac{2^{*}-2}{2\left(2^{*}-1\right)}$. Since $\left(v_{n}\right)$ is bounded in $H^{1}\left(\mathbb{R}^{N}\right), \int_{\mathbb{R}^{N}}\left|v_{n}-v\right|^{2} \rightarrow 0$ as $n \rightarrow \infty$. It follows from interpolation inequality that $\int_{\mathbb{R}^{N}}\left|v_{n}-v\right|^{q} \rightarrow 0$ for any $q \in\left[2,2^{*}\right)$. 
Step 3. $\left\|v_{n}-v\right\| \rightarrow 0$ as $n \rightarrow 0$.

Using $(2.1),\left(f_{5}\right),\left(f_{7}\right)$ and the Hölder's inequality, we have

$$
\begin{aligned}
& \left.\left|\int_{\mathbb{R}^{N}}\left(I_{\alpha} *\left|f\left(v_{n}^{+}\right)\right|^{p}\right)\right| f\left(v_{n}^{+}\right)\right|^{p-2} f\left(v_{n}^{+}\right) f^{\prime}\left(v_{n}^{+}\right)\left(v_{n}-v\right) \mid \\
\leq & C \int_{\mathbb{R}^{N}}\left(I_{\alpha} *\left|v_{n}\right|^{p / 2}\right)\left|v_{n}\right|^{\frac{p}{2}-1}\left|v_{n}-v\right| \\
\leq & C\left(\int_{\mathbb{R}^{N}}\left|v_{n}\right|^{\frac{p}{2} r}\right)^{1 / r}\left(\int_{\mathbb{R}^{N}}\left|v_{n}\right|^{\frac{p-2}{2} r}\left|v_{n}-v\right|^{r}\right)^{1 / r} \\
\leq & C\left(\left(\int_{\mathbb{R}^{N}}\left|v_{n}\right|^{\frac{p-2}{2} r \cdot \frac{p}{p-2}}\right)^{\frac{p-2}{p}}\left(\int_{\mathbb{R}^{N}}\left|v_{n}-v\right|^{r \cdot \frac{p}{2}}\right)^{2 / p}\right)^{1 / r} \\
\leq & C\left(\int_{\mathbb{R}^{N}}\left|v_{n}-v\right|^{r \cdot \frac{p}{2}}\right)^{2 /(p r)} \rightarrow 0,
\end{aligned}
$$

where $\frac{2}{r}-\frac{\alpha}{N}=1$. Since $\left\|I^{\prime}\left(v_{n}\right)\right\| \rightarrow 0$ and $\left\{v_{n}-v\right\}$ is bounded,

$$
\begin{aligned}
\left\langle I^{\prime}\left(v_{n}\right), v_{n}-v\right\rangle & =\int_{\mathbb{R}^{N}} \nabla v_{n} \nabla\left(v_{n}-v\right)+\int_{\mathbb{R}^{N}} V(x) f\left(v_{n}\right) f^{\prime}\left(v_{n}\right)\left(v_{n}-v\right) \\
& -\int_{\mathbb{R}^{N}}\left(I_{\alpha} *\left|f\left(v_{n}^{+}\right)\right|^{p}\right)\left|f\left(v_{n}^{+}\right)\right|^{p-2} f\left(v_{n}^{+}\right) f^{\prime}\left(v_{n}^{+}\right)\left(v_{n}-v\right) \rightarrow 0 .
\end{aligned}
$$

Further,

$$
\left|\int_{\mathbb{R}^{N}} V(x) f\left(v_{n}\right) f^{\prime}\left(v_{n}\right)\left(v_{n}-v\right)\right| \leq V_{\infty} \int_{\mathbb{R}^{N}}\left|v_{n}\right|\left|v_{n}-v\right| \rightarrow 0 .
$$

Hence

$$
\int_{\mathbb{R}^{N}} \nabla v_{n} \nabla\left(v_{n}-v\right) \rightarrow 0
$$

and

$$
\int_{\mathbb{R}^{N}}\left|\nabla\left(v_{n}-v\right)\right|^{2}=\int_{\mathbb{R}^{N}} \nabla v_{n} \nabla\left(v_{n}-v\right)-\int_{\mathbb{R}^{N}} \nabla v \nabla\left(v_{n}-v\right) \rightarrow 0 .
$$

From arguments above, we get that $\left\|v_{n}-v\right\| \rightarrow 0$ as $n \rightarrow \infty$.

Lemma 3.6 Up to a subsequence, $A:=\lim _{n \rightarrow \infty} \int_{\mathbb{R}^{N}}\left|f\left(v_{n}\right)\right|^{2}>0$.

Proof. We suppose, by contradiction, that $A=0$. By the Lemma 3.5, $v_{n} \rightarrow 0$ in $H^{1}\left(\mathbb{R}^{N}\right)$. Hence

$$
\int_{\mathbb{R}^{N}}\left(I_{\alpha} *\left|f\left(v_{n}^{+}\right)\right|^{p}\right)\left|f\left(v_{n}^{+}\right)\right|^{p} \leq C\left(\int_{\mathbb{R}^{N}}\left|v_{n}\right|^{\frac{p r}{2}}\right)^{\frac{2}{r}} \rightarrow 0
$$


where $\frac{2}{r}-\frac{\alpha}{N}=1$. Since

$$
\begin{aligned}
\left\langle I^{\prime}\left(v_{n}\right), \frac{f\left(v_{n}\right)}{f^{\prime}\left(v_{n}\right)}\right\rangle & =\int_{\mathbb{R}^{N}}\left(1+\frac{2 f^{2}\left(v_{n}\right)}{1+2 f^{2}\left(v_{n}\right)}\right)\left|\nabla v_{n}\right|^{2}+\int_{\mathbb{R}^{N}} V(x) f^{2}\left(v_{n}\right) \\
& -\int_{\mathbb{R}^{N}}\left(I_{\alpha} *\left|f\left(v_{n}^{+}\right)\right|^{p}\right)\left|f\left(v_{n}^{+}\right)\right|^{p} \rightarrow 0, \\
& \int_{\mathbb{R}^{N}}\left|\nabla v_{n}\right|^{2}+\int_{\mathbb{R}^{N}} V(x) f^{2}\left(v_{n}\right) \rightarrow 0 .
\end{aligned}
$$

It follows that

$$
c+o_{n}(1)=I\left(v_{n}\right)=\frac{1}{2}\left(\int_{\mathbb{R}^{N}}\left|\nabla v_{n}\right|^{2}+V(x) f^{2}\left(v_{n}\right)\right)-\frac{1}{2 p} \int_{\mathbb{R}^{N}}\left(I_{\alpha} *\left|f\left(v_{n}^{+}\right)\right|^{p}\right)\left|f\left(v_{n}^{+}\right)\right|^{p} \rightarrow 0,
$$

a contradiction. The proof is completed.

Lemma 3.7 Up to a subsequence, there exist $R, \beta>0$ and $\left\{x_{n}\right\} \subset \mathbb{R}^{N}$ such that

$$
\liminf _{n \rightarrow+\infty} \int_{B_{R}\left(x_{n}\right)}\left|v_{n}\right|^{2} \geq \beta
$$

Proof. By the Lemma 3.6, up to a subsequence, one has $A:=\lim _{n \rightarrow \infty} \int_{\mathbb{R}^{N}}\left|f\left(v_{n}\right)\right|^{2}>0$. If Lemma 3.7 is false, then it follows from the Lemma 1.21 in [26] that, up to a subsequence,

$$
v_{n} \rightarrow 0 \text { in } L^{2}\left(\mathbb{R}^{N}\right)
$$

Hence

$$
0<A=\lim _{n \rightarrow \infty} \int_{\mathbb{R}^{N}}\left|f\left(v_{n}\right)\right|^{2} \leq \lim _{n \rightarrow \infty} \int_{\mathbb{R}^{N}}\left|v_{n}\right|^{2}=0
$$

a contradiction. This completes the proof.

Lemma $3.8\left\langle I^{\prime}(v), \varphi\right\rangle=0$ for any $\varphi \in C_{0}^{\infty}\left(\mathbb{R}^{N}\right)$.

Proof. For any $\varphi \in C_{0}^{\infty}\left(\mathbb{R}^{N}\right)$, the support of $\varphi$ is contained in $B_{R_{0}}(0)$ for some $R_{0}>0$. Hence

$$
\begin{aligned}
& \left|\left\langle I^{\prime}\left(v_{n}\right)-I^{\prime}(v), \varphi\right\rangle\right| \\
\leq & \left|\int_{\mathbb{R}^{N}} \nabla\left(v_{n}-v\right) \nabla \varphi\right| \\
+ & \left|\int_{\mathbb{R}^{N}} V(x)\left(f\left(v_{n}\right) f^{\prime}\left(v_{n}\right)-f(v) f^{\prime}(v)\right) \varphi\right| \\
+ & \left|\int_{\mathbb{R}^{N}}\left[\left(I_{\alpha} *\left|f\left(v_{n}^{+}\right)\right|^{p}\right)\left|f\left(v_{n}^{+}\right)\right|^{p-1} f^{\prime}\left(v_{n}^{+}\right)-\left(I_{\alpha} *\left|f\left(v^{+}\right)\right|^{p}\right)\left|f\left(v^{+}\right)\right|^{p-1} f^{\prime}\left(v^{+}\right)\right] \varphi\right| \\
:= & I_{1}+I_{2}+I_{3} .
\end{aligned}
$$


For $I_{1}:=\left|\int_{\mathbb{R}^{N}} \nabla\left(v_{n}-v\right) \nabla \varphi\right|$, since $v_{n} \rightarrow v$ in $H^{1}\left(\mathbb{R}^{N}\right), I_{1} \rightarrow 0$ as $n \rightarrow \infty$.

For $I_{2}:=\left|\int_{\mathbb{R}^{N}} V(x)\left(f\left(v_{n}\right) f^{\prime}\left(v_{n}\right)-f(v) f^{\prime}(v)\right) \varphi\right|$, by $\left(f_{2}\right)$ and $\left(f_{3}\right)$, we have

$$
\left|f\left(v_{n}\right) f^{\prime}\left(v_{n}\right)-f(v) f^{\prime}(v)\right|^{2} \leq 2\left(\left|f\left(v_{n}\right) f^{\prime}\left(v_{n}\right)\right|^{2}+\left|f(v) f^{\prime}(v)\right|^{2}\right) \leq 2\left|v_{n}\right|^{2}+2|v|^{2} .
$$

By $v_{n} \rightarrow v$ in $L_{l o c}^{2}\left(\mathbb{R}^{N}\right)$ and the Lemma 3.4 , we obtain

$$
\lim _{n \rightarrow \infty} \int_{B_{R_{0}}(0)}\left|\left(f\left(v_{n}\right) f^{\prime}\left(v_{n}\right)-f(v) f^{\prime}(v)\right)\right|^{2}=0
$$

Using the Hölder inequality, we have

$$
\begin{aligned}
I_{2} & \leq V_{\infty} \int_{B_{R_{0}}(0)}\left|f\left(v_{n}\right) f^{\prime}\left(v_{n}\right)-f(v) f^{\prime}(v)\right||\varphi| \\
& \leq V_{\infty}\left(\int_{B_{R_{0}}(0)}\left|f\left(v_{n}\right) f^{\prime}\left(v_{n}\right)-f(v) f^{\prime}(v)\right|^{2}\right)^{\frac{1}{2}}\left(\int_{B_{R_{0}}(0)}|\varphi|^{2}\right)^{\frac{1}{2}} \rightarrow 0
\end{aligned}
$$

as $n \rightarrow \infty$.

Moreover,

$$
\begin{aligned}
I_{3}:= & \left|\int_{\mathbb{R}^{N}}\left[\left(I_{\alpha} *\left|f\left(v_{n}^{+}\right)\right|^{p}\right)\left|f\left(v_{n}^{+}\right)\right|^{p-1} f^{\prime}\left(v_{n}^{+}\right)-\left(I_{\alpha} *\left|f\left(v^{+}\right)\right|^{p}\right)\left|f\left(v^{+}\right)\right|^{p-1} f^{\prime}\left(v^{+}\right)\right] \varphi\right| \\
\leq & \int_{\mathbb{R}^{N}}\left(I_{\alpha} *\left|f\left(v_{n}^{+}\right)\right|^{p}\right)||\left|f\left(v_{n}^{+}\right)\right|^{p-1} f^{\prime}\left(v_{n}^{+}\right)-\left|f\left(v^{+}\right)\right|^{p-1} f^{\prime}\left(v^{+}\right)|| \varphi \mid \\
& +\left.\left|\int_{\mathbb{R}^{N}}\left(I_{\alpha} *\left|f\left(v_{n}^{+}\right)\right|^{p}\right)\right| f\left(v^{+}\right)\right|^{p-1} f^{\prime}\left(v^{+}\right) \varphi-\int_{\mathbb{R}^{N}}\left(I_{\alpha} *\left|f\left(v^{+}\right)\right|^{p}\right)\left|f\left(v^{+}\right)\right|^{p-1} f^{\prime}\left(v^{+}\right) \varphi \mid \\
& :=J_{1}+J_{2} .
\end{aligned}
$$

For $r=\frac{2 N}{N+\alpha}$, by $\left(f_{5}\right)$ and $\left(f_{7}\right)$,

$$
\begin{aligned}
& \left.|| f\left(v_{n}^{+}\right)\right|^{p-1} f^{\prime}\left(v_{n}^{+}\right)-\left.\left|f\left(v^{+}\right)\right|^{p-1} f^{\prime}\left(v^{+}\right)\right|^{r \cdot \frac{p}{p-2}} \\
& \leq C_{1}\left(\left.\left.|| f\left(v_{n}^{+}\right)\right|^{p-2} f\left(v_{n}^{+}\right) f^{\prime}\left(v_{n}^{+}\right)\right|^{r \cdot \frac{p}{p-2}}+\left.\left.|| f\left(v^{+}\right)\right|^{p-2} f\left(v^{+}\right) f^{\prime}\left(v^{+}\right)\right|^{r \cdot \frac{p}{p-2}}\right) \\
& \leq C_{2}\left(\left.\left.|| f^{2}\left(v_{n}^{+}\right)\right|^{\frac{p-2}{2}}\right|^{r \cdot \frac{p}{p-2}}+\left.\left.|| f^{2}\left(v^{+}\right)\right|^{\frac{p-2}{2}}\right|^{r \cdot \frac{p}{p-2}}\right) \\
& \leq C_{3}\left(\left|v_{n}\right|^{r \cdot \frac{p}{2}}+|v|^{r \cdot \frac{p}{2}}\right)
\end{aligned}
$$

Since $\frac{2(N+\alpha)}{N} \leq p<\frac{2(N+\alpha)}{N-2}, \frac{r p}{2} \in\left[2,2^{*}\right)$. By $v_{n} \rightarrow v$ in $L_{l o c}^{\frac{r p}{2}}\left(\mathbb{R}^{N}\right)$ and the Lemma 3.4 again, we obtain

$$
\left.\lim _{n \rightarrow \infty} \int_{B_{R_{0}}(0)}|| f\left(v_{n}^{+}\right)\right|^{p-1} f^{\prime}\left(v_{n}^{+}\right)-\left.\left|f\left(v^{+}\right)\right|^{p-1} f^{\prime}\left(v^{+}\right)\right|^{r \cdot \frac{p}{p-2}}=0 .
$$


By the boundedness of $\left(v_{n}\right)$, the Hölder inequality and (2.1), take $n \rightarrow \infty$,

$$
\begin{aligned}
J_{1} & =\int_{\mathbb{R}^{N}}\left(I_{\alpha} *\left|f\left(v_{n}^{+}\right)\right|^{p}\right)||\left|f\left(v_{n}^{+}\right)\right|^{p-1} f^{\prime}\left(v_{n}^{+}\right)-\left|f\left(v^{+}\right)\right|^{p-1} f^{\prime}\left(v^{+}\right)|| \varphi \mid \\
& \leq C_{1}\left(\int_{\mathbb{R}^{N}}\left|v_{n}\right|^{\frac{p}{2} r}\right)^{\frac{1}{r}}\left(\int_{\mathbb{R}^{N}}\left|f\left(v_{n}^{+}\right)\right|^{p-1} f^{\prime}\left(v_{n}^{+}\right)-\left.\left|f\left(v^{+}\right)\right|^{p-1} f^{\prime}\left(v^{+}\right)\right|^{r}|\varphi|^{r}\right)^{\frac{1}{r}} \\
& \leq C_{2}\left(\int_{B_{R_{0}}(0)}\left|f\left(v_{n}^{+}\right)\right|^{p-1} f^{\prime}\left(v_{n}^{+}\right)-\left.\left|f\left(v^{+}\right)\right|^{p-1} f^{\prime}\left(v^{+}\right)\right|^{r}|\varphi|^{r}\right)^{\frac{1}{r}} \\
& \leq C_{3}\left(\int_{B_{R_{0}}(0)}\left|f\left(v_{n}^{+}\right)\right|^{p-1} f^{\prime}\left(v_{n}^{+}\right)-\left.\left|f\left(v^{+}\right)\right|^{p-1} f^{\prime}\left(v^{+}\right)\right|^{r \cdot \frac{p}{p-2}}\right)^{\frac{p-2}{p r}}\left(\int_{B_{R_{0}}(0)}|\varphi|^{r \cdot \frac{p}{2}}\right)^{\frac{2}{p r}} \\
& \leq C_{4}\left(\int_{B_{R_{0}}(0)}\left|f\left(v_{n}^{+}\right)\right|^{p-1} f^{\prime}\left(v_{n}^{+}\right)-\left.\left|f\left(v^{+}\right)\right|^{p-1} f^{\prime}\left(v^{+}\right)\right|^{r \cdot \frac{p}{p-2}}\right)^{\frac{p-2}{p r}} \rightarrow 0
\end{aligned}
$$

where $r=\frac{2 N}{N+\alpha}$ is given in Remark 2.2-(2).

For $r=\frac{2 N}{N+\alpha}$, by $\frac{2(N+\alpha)}{N} \leq p<\frac{2(N+\alpha)}{N-2},\left(f_{7}\right)$ and the Hölder inequality, we have

$$
\begin{aligned}
\left.\left.\int_{\mathbb{R}^{N}}|| f\left(v^{+}\right)\right|^{p-1} f^{\prime}\left(v^{+}\right) \varphi\right|^{r} & \leq C \int_{\mathbb{R}^{N}}\left|f^{2}\left(v^{+}\right)\right|^{\frac{p-2}{2} \cdot r}|\varphi|^{r} \\
& \leq C \int_{\mathbb{R}^{N}}|v|^{\frac{p-2}{2} \cdot r}|\varphi|^{r} \\
& \leq C\left(\int_{\mathbb{R}^{N}}|v|^{\frac{p-2}{2} \cdot r \cdot \frac{p}{p-2}}\right)^{\frac{p-2}{p}}\left(\int_{\mathbb{R}^{N}}|\varphi|^{r \cdot \frac{p}{2}}\right)^{\frac{2}{p}} \\
& =C\left(\int_{\mathbb{R}^{N}}|v|^{\frac{p}{2} \cdot r}\right)^{\frac{p-2}{p}}\left(\int_{\mathbb{R}^{N}}|\varphi|^{r \cdot \frac{p}{2}}\right)^{\frac{2}{p}} \\
& =C|v|_{\frac{\frac{p r}{2}^{2}}{2}}^{\frac{p-2) r}{2}}|\varphi|_{\frac{p r}{2}}^{r} .
\end{aligned}
$$

It follows from $\frac{r p}{2} \in\left[2,2^{*}\right)$ that $\left|f\left(v^{+}\right)\right|^{p-1} f^{\prime}\left(v^{+}\right) \varphi \in L^{r}\left(\mathbb{R}^{N}\right)$.

In order to prove $J_{2} \rightarrow 0$, we use an argument which is partly an adaptation of the proof of the Proposition 2.2 in [18. Set a linear functional

$$
T(u):=\int_{\mathbb{R}^{N}}\left(I_{\alpha} * u\right)\left|f\left(v^{+}\right)\right|^{p-1} f^{\prime}\left(v^{+}\right) \varphi .
$$

Then, by $(2.1), T: L^{r}\left(\mathbb{R}^{N}\right) \rightarrow \mathbb{R}$, where $r=\frac{2 N}{N+\alpha}$, is a continuous linear functional, that is,

$$
|T(u)| \leq C\left(\int_{\mathbb{R}^{N}}|u|^{r}\right)^{\frac{1}{r}}\left(\left.\left.\int_{\mathbb{R}^{N}}|| f\left(v^{+}\right)\right|^{p-1} f^{\prime}\left(v^{+}\right) \varphi\right|^{r}\right)^{\frac{1}{r}} .
$$


As $\left(v_{n}\right)$ is bounded in $H^{1}\left(\mathbb{R}^{N}\right)$ and $\left|f\left(v_{n}^{+}\right)\right|^{p r} \leq\left|v_{n}\right|^{\frac{p r}{2}}$, the sequence $\left(\left|f\left(v_{n}^{+}\right)\right|^{p}\right)$ is bounded in $L^{r}\left(\mathbb{R}^{N}\right)$. We may assume, going if necessary to a subsequence, $\left|f\left(v_{n}^{+}\right)\right|^{p} \rightarrow\left|f\left(v^{+}\right)\right|^{p}$ in $L^{r}\left(\mathbb{R}^{N}\right)$. Then $T\left(\left|f\left(v_{n}^{+}\right)\right|^{p}\right) \rightarrow T\left(\left|f\left(v^{+}\right)\right|^{p}\right)$ as $n \rightarrow \infty$, that is,

$J_{2}=\left.\left|\int_{\mathbb{R}^{N}}\left(I_{\alpha} *\left|f\left(v_{n}^{+}\right)\right|^{p}\right)\right| f\left(v^{+}\right)\right|^{p-1} f^{\prime}\left(v^{+}\right) \varphi-\int_{\mathbb{R}^{N}}\left(I_{\alpha} *\left|f\left(v^{+}\right)\right|^{p}\right)\left|f\left(v^{+}\right)\right|^{p-1} f^{\prime}\left(v^{+}\right) \varphi \mid \rightarrow 0$.

So $I_{3}=J_{1}+J_{2} \rightarrow 0$ as $n \rightarrow \infty$. In a summary, up to a subsequence, we prove that $\left\langle I^{\prime}\left(v_{n}\right)-I^{\prime}(v), \varphi\right\rangle \rightarrow 0$ as $n \rightarrow \infty$. Since $\left\langle I^{\prime}\left(v_{n}\right), \varphi\right\rangle \rightarrow 0$, we have

$$
\left\langle I^{\prime}(v), \varphi\right\rangle=0
$$

Proof of Theorem 1.1 As a consequence of the Lemmas 3.1 and 3.2, for the constant

$$
c_{0}=\inf _{\gamma \in \Gamma} \sup _{t \in[0,1]} I(\gamma(t))>0
$$

where

$$
\Gamma=\left\{\gamma \in C\left([0,1], H^{1}\left(\mathbb{R}^{N}\right)\right): \gamma(0)=0, I(\gamma(1))<0\right\} .
$$

Hence, by the Theorem 6.3 in [29], there exists a Cerami sequence $\left(v_{n}\right)$ in $H^{1}\left(\mathbb{R}^{N}\right)$ at the level $c_{0}$, that is,

$$
I\left(v_{n}\right) \rightarrow c_{0} \text { and }\left(1+\left\|v_{n}\right\|\right)\left\|I^{\prime}\left(v_{n}\right)\right\| \rightarrow 0, \text { as } n \rightarrow \infty .
$$

By the Lemma 3.3, the sequence $\left\{v_{n}\right\}$ is bounded. Hence, up to a subsequence, one has $v_{n} \rightarrow v \in H^{1}\left(\mathbb{R}^{N}\right), v_{n}(x) \rightarrow v(x)$ a.e. $x \in \mathbb{R}^{N}$ and $v_{n} \rightarrow v$ in $L_{l o c}^{q}\left(\mathbb{R}^{N}\right)$ for all $q \in\left[2,2^{*}\right)$. Hence, by the Lemma 3.8, $\left\langle I^{\prime}(v), \varphi\right\rangle=0$ for any $\varphi \in C_{0}^{\infty}\left(\mathbb{R}^{N}\right)$, that is, $v$ is a weak solution of (1.3). We must prove that $v$ is nontrivial. For this, we follow the idea in [6], [15] and [4] to complete the proof of Theorem 1.1.

By the Lemma 3.7, up to a subsequence, there exist $R, \beta>0$ and $\left\{x_{n}\right\} \subset \mathbb{R}^{N}$ such that

$$
\liminf _{n \rightarrow+\infty} \int_{B_{R}\left(x_{n}\right)}\left|v_{n}\right|^{2} \geq \beta
$$

If $\left(V_{1}\right)$ holds, we may assume that $\left\{x_{n}\right\}$ is bounded. Then there exists $\rho>0$ such that $B_{R}\left(x_{n}\right) \subset B_{\rho}(0)$ for all $n$. Hence

$$
\int_{B_{\rho}(0)}|v|^{2}=\liminf _{n \rightarrow+\infty} \int_{B_{\rho}(0)}\left|v_{n}\right|^{2} \geq \liminf _{n \rightarrow+\infty} \int_{B_{R}\left(x_{n}\right)}\left|v_{n}\right|^{2} \geq \beta>0 .
$$


It follows that $v$ is nontrivial. It is easy to see that $v \geq 0$ in $\mathbb{R}^{N}$. Hence $v \in H^{1}\left(\mathbb{R}^{N}\right)$ is a nontrivial, nonnegative, weak solution of Eq. (1.3). By the Lemma 2.5, $v>0$ in $\mathbb{R}^{N}$.

If $\left(V_{2}\right)$ holds, we assume, by contradiction, that $v \equiv 0$. Consider the following two limit functionals:

$$
I_{\infty}(v)=\frac{1}{2} \int_{\mathbb{R}^{N}}\left(|\nabla v|^{2}+V_{\infty} f^{2}(v)\right)-\frac{1}{2 p} \int_{\mathbb{R}^{N}}\left(I_{\alpha} *\left|f\left(v^{+}\right)\right|^{p}\right)\left|f\left(v^{+}\right)\right|^{p}
$$

and

$$
J_{\infty}(u)=\frac{1}{2} \int_{\mathbb{R}^{N}}\left[\left(1+2 u^{2}\right)|\nabla u|^{2}+V_{\infty} u^{2}\right]-\frac{1}{2 p} \int_{\mathbb{R}^{N}}\left(I_{\alpha} *\left|u^{+}\right|^{p}\right)\left|u^{+}\right|^{p},
$$

where $u=f(v)$. Define

$$
c_{\infty}=\inf _{\gamma \in \Gamma} \sup _{t \in[0,1]} I_{\infty}(\gamma(t))>0
$$

where

$$
\Gamma=\left\{\gamma \in C\left([0,1], H^{1}\left(\mathbb{R}^{N}\right)\right): \gamma(0)=0, I_{\infty}(\gamma(1))<0\right\}
$$

Notice that $V_{0}<V_{\infty}$, we have

$$
c_{0}<c_{\infty}
$$

To complete the proof of Theorem 1.1, we divide into the following four lemmas.

Lemma $3.9 I_{\infty}\left(v_{n}\right) \rightarrow c_{0}$ and $\left(1+\left\|v_{n}\right\|\right)\left\|I_{\infty}^{\prime}\left(v_{n}\right)\right\| \rightarrow 0$, as $n \rightarrow \infty$.

Proof. Notice that $\left(v_{n}\right)$ is bounded in $H^{1}\left(\mathbb{R}^{N}\right)$, there exists $M_{1}>0$ such that $M_{1}>$ $2 V_{\infty}$ and $M_{1}>\int_{\mathbb{R}^{N}} f^{2}\left(v_{n}\right)$. Since $v_{n} \rightarrow v=0$ in $L_{l o c}^{q}\left(\mathbb{R}^{N}\right)$ for all $q \in\left[2,2^{*}\right)$ and $V(x) \leq$ $V_{\infty}:=\lim _{|y| \rightarrow \infty} V(y)<\infty$ for all $x \in \mathbb{R}^{N}$. For every $\epsilon>0$, there is $M>0$ such that, for $n$ large enough, one has

$$
0 \leq V_{\infty}-V(x)<\frac{\epsilon}{2 M_{1}}, \quad \forall|x| \geq M
$$

and

$$
\int_{B_{M}(0)}\left|v_{n}\right|^{2}<\frac{\epsilon}{4 V_{\infty}} .
$$


Hence

$$
\begin{aligned}
0 & \leq \int_{\mathbb{R}^{N}} V_{\infty} f^{2}\left(v_{n}\right)-\int_{\mathbb{R}^{N}} V(x) f^{2}\left(v_{n}\right) \\
& =\int_{\mathbb{R}^{N} \backslash B_{M}(0)}\left(V_{\infty}-V(x)\right) f^{2}\left(v_{n}\right)+\int_{B_{M}(0)}\left(V_{\infty}-V(x)\right) f^{2}\left(v_{n}\right) \\
& <M_{1} \cdot \frac{\epsilon}{2 M_{1}}+2 V_{\infty} \cdot \frac{\epsilon}{4 V_{\infty}} \\
& =\epsilon
\end{aligned}
$$

and

$$
\left|I_{\infty}\left(v_{n}\right)-I\left(v_{n}\right)\right|=\int_{\mathbb{R}^{N}} V_{\infty} f^{2}\left(v_{n}\right)-\int_{\mathbb{R}^{N}} V(x) f^{2}\left(v_{n}\right) \rightarrow 0
$$

as $n \rightarrow+\infty$. Similarly,

$$
\begin{aligned}
\left\|I_{\infty}^{\prime}\left(v_{n}\right)-I^{\prime}\left(v_{n}\right)\right\| & =\sup _{\|\phi\|=1}\left|\left\langle I_{\infty}^{\prime}\left(v_{n}\right)-I^{\prime}\left(v_{n}\right), \phi\right\rangle\right| \\
& =\sup _{\|\phi\|=1}\left|\int_{\mathbb{R}^{N}}\left(V_{\infty}-V(x)\right) f\left(v_{n}\right) f^{\prime}\left(v_{n}\right) \phi\right| \rightarrow 0
\end{aligned}
$$

as $n \rightarrow+\infty$.

It follows that $\left\{v_{n}\right\}$ is also a Cerami sequence of $I_{\infty}$ at the level $c_{0}$.

Lemma 3.10 Let $p>2, a>0, b \geq 0, c>0$ and $h(t):=a+b t^{2}-c t^{2 p-2}$ for $t \geq 0$. Then there exists a unique $t_{0}>0$ such that

$$
h\left(t_{0}\right)=0, h(t)>0 \text { for } 0 \leq t<t_{0} \text { and } h(t)<0 \text { for } t>t_{0} .
$$

The proof of Lemma 3.10 is standard.

Lemma 3.11. Let $v_{0} \in H^{1}\left(\mathbb{R}^{N}\right), u_{0}=f\left(v_{0}\right)$ such that

$$
\begin{gathered}
a:=\int_{\mathbb{R}^{N}}\left|\nabla u_{0}\right|^{2}+\int_{\mathbb{R}^{N}} V_{\infty} u_{0}^{2}>0, \\
b:=4 \int_{\mathbb{R}^{N}} u_{0}^{2}\left|\nabla u_{0}\right|^{2}>0
\end{gathered}
$$

and

$$
c:=\int_{\mathbb{R}^{N}}\left(I_{\alpha} *\left|u_{0}^{+}\right|^{p}\right)\left|u_{0}^{+}\right|^{p}>0 .
$$

Then there exist $t_{1}>t_{0}>0$ such that

$$
J_{\infty}\left(t_{0} u_{0}\right)>J_{\infty}\left(t u_{0}\right), \quad \forall t \in[0,+\infty) \backslash\left\{t_{0}\right\}
$$


and

$$
J_{\infty}\left(t_{1} u_{0}\right)<0
$$

Proof. By the definitions of $I_{\infty}(v)$ and $J_{\infty}(u)$, we know that

$$
I_{\infty}\left(v_{0}\right)=J_{\infty}\left(u_{0}\right)=\frac{1}{2} \int_{\mathbb{R}^{N}}\left[\left(1+2 u_{0}^{2}\right)\left|\nabla u_{0}\right|^{2}+V_{\infty} u_{0}^{2}\right]-\frac{1}{2 p} \int_{\mathbb{R}^{N}}\left(I_{\alpha} *\left|u_{0}^{+}\right|^{p}\right)\left|u_{0}^{+}\right|^{p}
$$

Set

$$
g(t)=J_{\infty}\left(t u_{0}\right)=\frac{1}{2} \int_{\mathbb{R}^{N}}\left[\left(1+2 t^{2} u_{0}^{2}\right)\left|\nabla\left(t u_{0}\right)\right|^{2}+V_{\infty} t^{2} u_{0}^{2}\right]-\frac{1}{2 p} \int_{\mathbb{R}^{N}}\left(I_{\alpha} *\left|t u_{0}^{+}\right|^{p}\right)\left|t u_{0}^{+}\right|^{p} .
$$

Then

$$
\frac{d}{d t}(g(t))=t\left(a+b t^{2}-c t^{2 p-2}\right) .
$$

By $p>2$ and the Lemma 3.10, there is an unique $t_{0}>0$ such that $g^{\prime}\left(t_{0}\right)=0, g^{\prime}(t)>0$ for $0<t<t_{0}$ and $g^{\prime}(t)<0$ for $t>t_{0}$. Hence

$$
J_{\infty}\left(t_{0} u_{0}\right)>J_{\infty}\left(t u_{0}\right), \quad \forall t \in[0,+\infty) \backslash\left\{t_{0}\right\}
$$

Since $p>2$, it is easy to see that

$$
J_{\infty}\left(t u_{0}\right) \rightarrow-\infty \text { as } t \rightarrow+\infty .
$$

Therefore, there exists $t_{1}>t_{0}$ such that

$$
J_{\infty}\left(t_{1} u_{0}\right)<0
$$

Remark 3.12 Set

$$
\gamma_{0}(t)=f^{-1}\left(t t_{1} u_{0}\right), t \in[0,1] .
$$

Then

$$
I_{\infty}\left(\gamma_{0}(t)\right)=J_{\infty}\left(t t_{1} u_{0}\right) \leq J_{\infty}\left(t_{0} u_{0}\right)=J_{\infty}\left(\frac{t_{0}}{t_{1}} \cdot t_{1} u_{0}\right)=I_{\infty}\left(\gamma_{0}\left(\frac{t_{0}}{t_{1}}\right)\right), \forall t \in[0,1] .
$$

It follows that

$$
c_{\infty}=\inf _{\gamma \in \Gamma} \sup _{t \in[0,1]} I_{\infty}(\gamma(t)) \leq \sup _{t \in[0,1]} I_{\infty}\left(\gamma_{0}(t)\right)=I_{\infty}\left(\gamma_{0}\left(\frac{t_{0}}{t_{1}}\right)\right)=J_{\infty}\left(t_{0} u_{0}\right) .
$$


Lemma $3.13 c_{\infty} \leq c_{0}$.

Proof. By the Lemma 3.6 we have $A:=\lim _{n \rightarrow \infty} \int_{\mathbb{R}^{N}}\left|f\left(v_{n}\right)\right|^{2}>0$. Notice that

$$
\left\langle I_{\infty}^{\prime}\left(v_{n}\right), \frac{f\left(v_{n}\right)}{f^{\prime}\left(v_{n}\right)}\right\rangle=o_{n}(1) .
$$

Passing to a subsequence, for $n$ large enough, we get

$$
\begin{aligned}
0 & <\frac{1}{2} V_{\infty} A \\
& \leq V_{\infty} \int_{\mathbb{R}^{N}}\left|f\left(v_{n}\right)\right|^{2} \\
& =\int_{\mathbb{R}^{N}} V_{\infty}\left|f\left(v_{n}\right)\right|^{2} \\
& \leq\left\langle I_{\infty}^{\prime}\left(v_{n}\right), \frac{f\left(v_{n}\right)}{f^{\prime}\left(v_{n}\right)}\right\rangle+\int_{\mathbb{R}^{N}}\left(I_{\alpha} *\left|f\left(v_{n}^{+}\right)\right|^{p}\right)\left|f\left(v_{n}^{+}\right)\right|^{p} \\
& =o_{n}(1)+\int_{\mathbb{R}^{N}}\left(I_{\alpha} *\left|f\left(v_{n}^{+}\right)\right|^{p}\right)\left|f\left(v_{n}^{+}\right)\right|^{p}
\end{aligned}
$$

for $n$ large enough. Hence

$$
\int_{\mathbb{R}^{N}}\left(I_{\alpha} *\left|f\left(v_{n}^{+}\right)\right|^{p}\right)\left|f\left(v_{n}^{+}\right)\right|^{p} \geq \frac{1}{3} V_{\infty} A>0
$$

for large $n$. By $\left\langle I_{\infty}^{\prime}\left(v_{n}\right), \frac{f\left(v_{n}\right)}{f^{\prime}\left(v_{n}\right)}\right\rangle=o_{n}(1)$ again, we have

$$
\int_{\mathbb{R}^{N}}\left(1+\frac{2 f^{2}\left(v_{n}\right)}{1+2 f^{2}\left(v_{n}\right)}\right)\left|\nabla v_{n}\right|^{2}+\int_{\mathbb{R}^{N}} V_{\infty} f^{2}\left(v_{n}\right)-\int_{\mathbb{R}^{N}}\left(I_{\alpha} *\left|f\left(v_{n}^{+}\right)\right|^{p}\right)\left|f\left(v_{n}^{+}\right)\right|^{p}=o_{n}(1) .
$$

Set $u_{n}:=f\left(v_{n}\right)$. Then

$$
\int_{\mathbb{R}^{N}}\left(1+4 u_{n}^{2}\right)\left|\nabla u_{n}\right|^{2}+\int_{\mathbb{R}^{N}} V_{\infty} u_{n}^{2}-\int_{\mathbb{R}^{N}}\left(I_{\alpha} *\left|u_{n}^{+}\right|^{p}\right)\left|u_{n}^{+}\right|^{p}=o_{n}(1) .
$$

Put $a_{n}:=\int_{\mathbb{R}^{N}}\left|\nabla u_{n}\right|^{2}+\int_{\mathbb{R}^{N}} V_{\infty} u_{n}^{2}, \quad b_{n}:=4 \int_{\mathbb{R}^{N}} u_{n}^{2}\left|\nabla u_{n}\right|^{2}$ and $c_{n}:=\int_{\mathbb{R}^{N}}\left(I_{\alpha} *\left|u_{n}^{+}\right|^{p}\right)\left|u_{n}^{+}\right|^{p}$. Then

$$
a_{n}+b_{n}-c_{n}=o_{n}(1) \text {. }
$$

Furthermore, since $\left(u_{n}\right)$ is bounded in $H^{1}\left(\mathbb{R}^{N}\right),\left(a_{n}\right),\left(b_{n}\right),\left(c_{n}\right)$ are all bounded. Hence, passing to a subsequence, we can assume that there are $a, b, c \in[0,+\infty)$ such that $a_{n} \rightarrow$ $a, b_{n} \rightarrow b, c_{n} \rightarrow c$ as $n \rightarrow+\infty$ and $a+b-c=0$. Moreover, for $n$ large enough, one has

$$
c_{n}=\int_{\mathbb{R}^{N}}\left(I_{\alpha} *\left|u_{n}^{+}\right|^{p}\right)\left|u_{n}^{+}\right|^{p}=\int_{\mathbb{R}^{N}}\left(I_{\alpha} *\left|f\left(v_{n}^{+}\right)\right|^{p}\right)\left|f\left(v_{n}^{+}\right)\right|^{p} \geq \frac{1}{3} V_{\infty} A>0
$$


and

$$
a_{n}=\int_{\mathbb{R}^{N}}\left|\nabla u_{n}\right|^{2}+\int_{\mathbb{R}^{N}} V_{\infty} u_{n}^{2} \geq \int_{\mathbb{R}^{N}} V_{\infty} f\left(v_{n}\right)^{2} \geq \frac{1}{2} V_{\infty} A>0,
$$

It follows that $a>0, c>0$. It follows from the Lemma 3.10 that there exists a unique sequence $\left(t_{n}\right) \subset(0,+\infty)$ such that $a_{n}+b_{n} t_{n}^{2}-c_{n} t_{n}^{2 p-2}=0$. Since $c>0,\left(t_{n}\right)$ is bounded. We may assume that there is $t \geq 0$ such that $t_{n} \rightarrow t$. Then, $a+b t^{2}-c t^{2 p-2}=0$. Since $a+b-c=0$, by Lemma 3.10 again, we get $t=1$. By the Lemma 3.11,

$$
J_{\infty}\left(t_{n} u_{n}\right)>J_{\infty}\left(t u_{n}\right), \forall t \in[0,+\infty) \backslash\left\{t_{n}\right\} .
$$

Hence $c_{\infty} \leq J_{\infty}\left(t_{n} u_{n}\right)$ by Remark 3.12. Further,

$$
\begin{aligned}
J_{\infty}\left(t_{n} u_{n}\right)-I_{\infty}\left(v_{n}\right) & =J_{\infty}\left(t_{n} u_{n}\right)-J_{\infty}\left(u_{n}\right) \\
& =\frac{1}{2} \int_{\mathbb{R}^{N}}\left[\left(1+2 t_{n}^{2} u_{n}^{2}\right)\left|\nabla\left(t_{n} u_{n}\right)\right|^{2}+V_{\infty} t_{n}^{2} u_{n}^{2}\right] \\
& -\frac{1}{2 p} \int_{\mathbb{R}^{N}}\left(I_{\alpha} *\left|t_{n} u_{n}^{+}\right|^{p}\right)\left|t_{n} u_{n}^{+}\right|^{p} \\
& -\frac{1}{2} \int_{\mathbb{R}^{N}}\left[\left(1+2 u_{n}^{2}\right)\left|\nabla\left(u_{n}\right)\right|^{2}+V_{\infty} u_{n}^{2}\right] \\
& +\frac{1}{2 p} \int_{\mathbb{R}^{N}}\left(I_{\alpha} *\left|u_{n}^{+}\right|^{p}\right)\left|u_{n}^{+}\right|^{p} \\
& =\frac{1}{2} a_{n}\left(t_{n}^{2}-1\right)+\frac{1}{4} b_{n}\left(t_{n}^{4}-1\right)-\frac{1}{2 p} c_{n}\left(t_{n}^{2 p}-1\right) \\
& =o_{n}(1) .
\end{aligned}
$$

Hence

$$
c_{\infty} \leq J_{\infty}\left(t_{n} u_{n}\right)=I_{\infty}\left(v_{n}\right)+o_{n}(1)
$$

So,

$$
c_{\infty} \leq c_{0}
$$

Contrasting the Lemma 3.13 and (3.2), we get a contradiction. It shows that $v$ is nontrivial. As the case $\left(V_{1}\right)$, we know $v>0$. This completes the proof of Theorem 1.1. 


\section{References}

[1] C. O. Alves, A. B. Nbrega, M. Yang, Multi-bump solutions for Choquard equation with deepening potential well, Calculus of Variations, 55(2015), 1-28.

[2] C. O. Alves and M. Yang, Multiplicity and concentration of solutions for a quasilinear Choquard equation, J. Math. Phys. 55(2014), 423-443.

[3] Y. Chen and X. Wu, Existence of nontrivial solutions and high energy solutions for a class of quasilinear Schrödinger equations via the dual-perturbation method, Abstract and Applied Analysis, (2013), DOI: 10. 1155/2013/256324.

[4] M. Colin, L. Jeanjean, Solutions for a quasilinear Schrödinger equation: a dual approach, Nonlinear Anal. 56(2004), 213-226.

[5] J. M. do Ó, O. H. Miyagaki, S. H. M. Soares, Soliton solutions for quasilinear Schrödinger equations: the critical exponential case, Nonlinear Anal. 67(2007), 33573372 .

[6] J. M. do Ó, O. H. Miyagaki, S. H. M. Soares, Soliton solutions for quasilinear Schrödinger equations with critical growth, J. Differ. Equation. 248(2010), 722-744.

[7] X. Fan, A constrained minimization problem involving the $\mathrm{p}(\mathrm{x})$-Laplacian in $\mathbb{R}^{N}$, Nonlinear Anal. 69(2008), 3661-3670.

[8] X. Fang and A. Szulkin, Multiple solutions for a quasilinear Schrödinger equation, J. Differ. Equation. 254(2013), 2015-2032.

[9] D. Gilbargand N. S. Trudinger, Elliptic partial differential equations of second order, Springer, 1997.

[10] S. Kurihura, Large-amplitude quasi-solitons in superfluid films, J. Phys. Soc. Jpn. 50(1981), 3262-3267.

[11] E. W. Laedke, K. H. Spatschek, L. Stenflo, Evolution theorem for a class of perturbed envelope soliton solutions, J. Math. Phys. 24(1983), 2764-2769. 
[12] S. Li, J. Xiang and X. Zeng, Ground states of nonlinear Choquard equations with multi-well potentials, J. Math. Phys. 57, 081515(2016), doi: 10.1063/1.4961158.

[13] A. G. Litvak, A. M. Sergeev, One dimensional collapse of plasma waves, JETP Lett. $27(1978), 517-520$.

[14] J. Liu, Z. Q. Wang, Soliton solutions for quasilinear Schrödinger equations: I, Proc. Am. Math. Soc. 131(2003), 441-448.

[15] J. Liu, Y. Wang, Z. Q. Wang, Soliton solutions for quasilinear Schrödinger equations: II, J. Differ. Equation. 187(2003), 473-493.

[16] J. Liu, Y. Wang, Z. Q. Wang, Solutions for quasilinear Schrödinger equations via the Nehari method, Commun. Partial Diff. Eqns. 29(2004), 879-901.

[17] V. Moroz and J. Van Schaftingen, Groundstates of nonlinear Choquard equations: existence, qualitative properties and decay asymptotics, J. Funct. Anal. 265(2013), 153-184.

[18] V. Moroz and J. Van Schaftingen, Existence of groundstates for a class of nonlinear Choquard equations, Trans. Am. Math. Soc. 367(2015), 6557-6579.

[19] A. Nakamura, Damping and modification of exciton solitary waves, J. Phys. Soc. Jpn. 42(1977), 1824-1835.

[20] M. Poppenberg, On the local well posedness of quasi-linear Schrödinger equations in arbitrary space dimension, J. Differ. Equation. 172(2001), 83-115.

[21] M. Poppenberg, K. Schmitt, Z. Q. Wang, On the existence of soliton solutions to quasilinear Schrödinger equations, Calc. Var. 14(2002), 329-344.

[22] M. Porkolab, M. V. Goldman, Upper hybrid solitons and oscillating two-stream instabilities, Phys. Fluids. 19(1976), 872-881.

[23] D. Ruiz, G. Siciliano, Existence of ground states for a modified nonlinear Schrödinger equation, Nonlinearity, 23(2010), 1221-1233. 
[24] J. Seok, Nonlinear Choquard equations involving a critical local term, Applied Mathematics Letters, 63(2017),77-87.

[25] M. Struwe, Variational methods: Applications to nonlinear partial differential equations and Hamiltonian systems, 4th ed., Springer, 2008.

[26] M. Willem, Minimax Theorems, Birkhäuser, Boston, 1996.

[27] Z. Wu, J. Yin and C. Wang, Elliptic and Parabolic Equations, World Scientific, Singapore, 2006.

[28] H. Ye, Mass minimizers and concentration for nonlinear Choquard equations in $\mathbb{R}^{N}$, Mathematics, 48(2016), 393-417.

[29] C. Zhong, X. Fan, W. Chen, Introduction of Non-linear Functional Analysis, Lanzhou University Publishing House, 1998. 